\title{
Emergency coronary artery stenting for coronary dissection complicating diagnostic cardiac catheterisation
}

\author{
Charles Knight, Rod Stables, Ulrich Sigwart
}

\begin{abstract}
Dissection of a coronary artery is a recognised and significant complication of diagnostic coronary angiography that often requires emergency coronary artery bypass grafting. A coronary stent was used to treat this complication. This technique has potential advantages in terms of speed of reperfusion and availability in centres performing diagnostic angiography without surgical cover on site.
\end{abstract}

(Br Heart f 1995;74:199-201)

Keywords: stenting, dissection, coronary artery, cardiac catheterisation

\section{Case Report}

The patient, a 48 year old bus driver, holding a public service vehicle licence, first presented in 1987 when his general practitioner noted a murmur and referred him for cardiological assessment. Examination at that time showed signs of mild mixed aortic valve disease. The electrocardiogram and chest $\mathrm{X}$ ray were both normal. Doppler echocardiography showed good left ventricular systolic function with left ventricular hypertrophy. There was a systolic pressure difference of $20 \mathrm{~mm} \mathrm{Hg}$ across the aortic valve and mild aortic regurgitation was detected. The patient was advised about antibiotic prophylaxis and a recommendation was made that he could continue to hold his public service vehicle licence subject to regular outpatient review. He remained symptom free. Repeat echocardiography in 1992 showed an increase in the peak aortic valve gradient to $50 \mathrm{~mm} \mathrm{Hg}$, with moderate aortic regurgitation. Cardiac catheterisation was undertaken.

The right femoral approach was used. The coronary arteries were normal. The aortic valve was crossed after several attempts with a 0.038 inch soft-tipped straight wire and right Judkins 4 catheter. The systolic pressure difference across the valve on withdrawal was $16 \mathrm{~mm} \mathrm{Hg}$. Immediately after withdrawal, chest pain developed and the patient became bradycardic and hypotensive. The electrocardiogram showed ST elevation in the inferior leads. A temporary pacing wire was inserted. Injection of contrast into the right coronary artery showed proximal occlusion and extensive dissection of the artery (fig B), presumably caused by injury by the guide wire during attempts to cross the valve. It was decided to attempt to reopen the artery immediately by angioplasty. A 0.014 inch Entre guide wire (Scimed) was passed down the true lumen of the artery and a $3.5 \mathrm{~mm}$ Speedflow perfusion balloon (Schneider) was inflated proximally. This failed to restore lasting anterograde flow. We therefore decided to proceed to stenting. A Palmaz-Schatz stent (PS 153) was deployed, using the same balloon as a delivery device, in the proximal artery to cover the presumed dissection entry site. This procedure immediately restored vessel patency, although there was some residual narrowing, presumably intramural haematoma, in the mid-vessel. The chest pain and ST elevation in the inferior leads were abolished within 15 minutes of the dissection.

The patient was well after the procedure and was fully anticoagulated with heparin, aspirin, dipyridamole, and warfarin. There was a small rise in cardiac enzymes (peak creatine kinase $M B$ of 71 units/l) and $R$ wave amplitude in the inferior leads was slightly reduced. Repeat angiography after 24 hours showed the vessel to be widely patent with excellent flow (fig C). The intramural haematoma that had been present distal to the dissection site had completely resolved. The patient made a good recovery. Five days after the procedure he had two short episodes of chest pain associated with changes in the inferior leads of the electrocardiogram. He was treated with both thrombolysis and calcium antagonists to cover the possibilities of thrombus formation around the stent or vessel spasm. The rapid resolution of the pain on each occasion suggested vessel spasm and the pain did not recur. The patient underwent limited exercise testing before discharge. He completed stage VI of the modified Bruce protocol without chest pain developing. Anticoagulation was stopped after 3 months. On subsequent outpatient review he was well.

\section{Discussion}

Coronary dissection is a recognised and potentially serious complication of coronary angiography. A recent confidential enquiry into complications of diagnostic catheterisation in 34041 patients in the United 
Right coronary artery before dissection $(A)$, after dissection (B) and 24 hours after stent deployment (with stent position marked) (C).
A
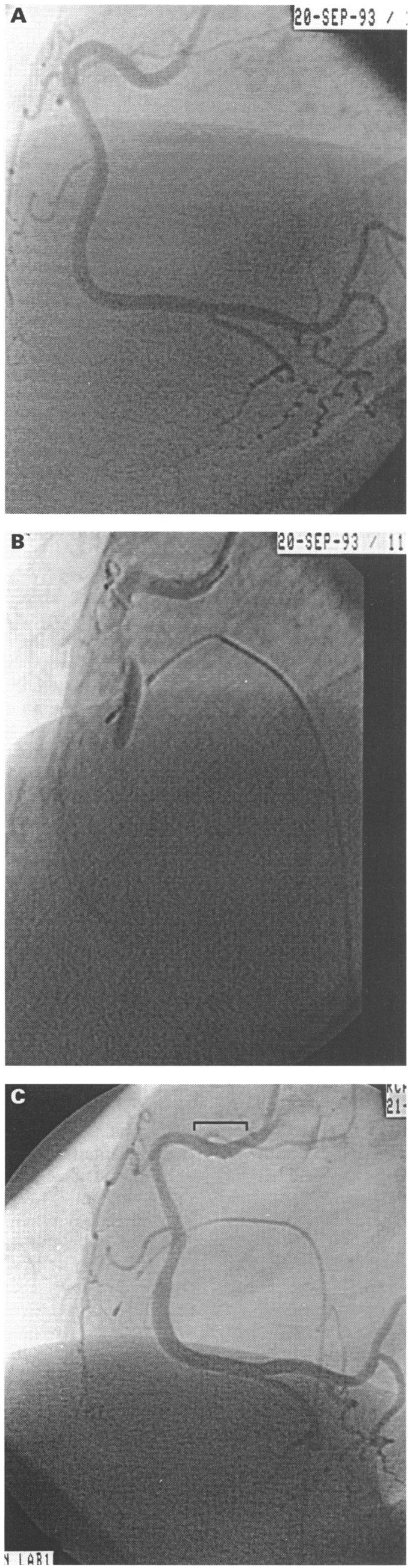

Kingdom reported 20 cases of coronary dissection, an incidence of $0.06 \% .^{1}$ Fourteen of these caused significant ischaemia. The mortality of coronary dissection in this series was $15 \%$, and $7 \%$ of all deaths complicating angiography were caused by dissection. Most of these cases probably occurred as a result of catheter-induced ostial trauma during coronary angiography. There is some evidence that dissection is more common with smaller $6 \mathrm{~F}$ catheters $(0.67 \% v 0.04 \%$ for $8 \mathrm{~F}$ catheters in one report of 3006 patients undergoing diagnostic angiography ${ }^{2}$ ). In the case we present, it is likely that the dissection was caused not by the catheter at angiography but by the guide wire during attempts to cross the aortic valve. The risk of such a complication may by minimised if crossing the aortic valve with a guide wire is attempted only by experienced operators: if the valve is not crossed easily the trans-septal approach should be considered.

The ideal management of acute coronary occlusion, whatever the cause, is the prompt restoration of vessel patency to limit the extent and duration of ischaemia. In the setting of dissection during angiography the usual approach to myocardial salvage has been urgent referral for coronary artery bypass grafting. In the confidential enquiry report quoted above, $60 \%$ of patients were treated in this way. ${ }^{1}$ The main disadvantages of this approach are the associated morbidity and the delay in arranging emergency surgery. In centres without surgical cover on site the delay will be considerable.

Coronary stenting has an established role in the treatment of coronary artery disease both as a primary procedure and in the management of complications arising from percutaneous transluminal coronary angioplasty. ${ }^{34}$ It has also been used for abrupt vessel closure during coronary angioplasty as a temporary bridge to surgery. ${ }^{5}$ Much thought has been given to the choice of stents and the required length of the device. The exact entry site of the dissection is often difficult to determine in patients with coronary artery disease and a longer stent or several stents are required to achieve the best outcome, although even in this situation, there is evidence that residual coronary dissection, not completely covered by the stent, has resolved in most patients at follow up angiography. ${ }^{6}$ In the presence of an iatrogenic dissection in an otherwise healthy vessel the entry point of the dissection may be better defined. Although we were unable to see the exact entry site angiographically, it could be presumed with a degree of certainty and we decided not to continue with further stent implants despite the initially unsatisfactory appearance of the distal vessel caused by an intramural haematoma. The haematoma cleared after 24 hours.

This is the first case, to our knowledge, in which coronary stenting was used instead of coronary artery surgery to manage iatrogenic coronary dissection during diagnostic angiography. This approach has clear advantages in terms of speed of reperfusion; in our patient 
flow was restored within 15 minutes. Stenting also avoids surgery, in patients who would otherwise not require it. In centres without surgical cover on site this approach could be life saving.

We thank Professor Poole-Wilson for his permission to report the patient.

1 De Bono D. Complications of diagnostic cardiac catheterisation: results from 34041 patients in the United Kingdom confidential enquiry into cardiac catheter complications. Br Heart $₹$ 1993;70:297-300.

2 Prewitt KC, Zen B, Wortham DC, Pearson C. Increased risk of coronary artery dissection during coronary angiography with $6 \mathrm{~F}$ catheters. Angiology 1993;44:107-13

3 Sigwart U, Puel J, Mirkovitch V, Joffre F, Kappenberger L. Intravascular stents to prevent occlusion and restenosis after transluminal angioplasty. $N$ Engl $\mathcal{F}$ Med 1987;316: $701-6$.

4 Sigwart U, Urban P, Golf S, Kaufmann U, Imbert C, Fischer A et al. Emergency stenting for acute occlusion after coronary balloon angioplasty. Circulation 1988;78: after coron.

5 Chronos N, Stables R, Gibbs S, Nordrehaug JE, Sigwart $\mathrm{U}$, Buller $\mathrm{N}$. Case report: temporary stenting as a bridge to surgery. $\mathcal{F}$ Intervent Cardiol 1994;7:327-30.

6 Alfonso F, Hernandez R, Goicolea J, Segovia J, PerezVizcayno MJ, Banuelos C, et al. Coronary stenting for acute coronary dissection after coronary angioplasty: implications of the residual dissection. $\mathcal{f} \mathrm{Am}$ Coll Cardiol 1994;24:989-96. 